\title{
Application of the gross theory to FP decay heat calculations in the new light of TAGS measurements
}

\author{
Tadashi Yoshida ${ }^{1}$, Yuuji Wakasugi ${ }^{1}$, Naoto Hagura ${ }^{1}$, and Takahiro Tachibana ${ }^{2}$ \\ 1 Musashi Institute of Technology, Tokyo 158-8557, Japan \\ 2 Senior High School of Waseda University, Tokyo 177-0044, Japan
}

\begin{abstract}
On the basis of the recent knowledge that the decay data measured by Total Absorption Gamma-ray Spectrometer (TAGS) is free from the so-called pandemonium, the prediction power of the gross theory of beta-decay was studied. By comparing the theoretical prediction with the INEL/TAGS data, we conclude that the gross theory predicts well the total energy release, or $E_{\beta}+E_{\gamma}$. However, when $E_{\beta}$ and $E_{\gamma}$ are dealt with separately, the prediction power of the theory has a limitation. This is one of the reasons why we need further TAGS measurements.
\end{abstract}

\section{Introduction}

In the early stage of development of the fission product (FP) decay data libraries around 1980, a serious inconsistency between calculations based on these libraries and sampleirradiation measurements was experienced in Japan, in the US and also in Europe. This problem was practically circumvented by introduction of nuclear model calculations [1] in the cases of the JENDL FP Decay Data File and ENDF-B/VI. This type of inconsistency between summation calculations and the experiments was proved to be originated from so-called pandemonium problem [2]. Recently it was shown that the use of the experimental results based on the total absorption gamma-ray spectrometer (TAGS) improve the situation fairly well without introducing any theoretical prediction [3]. TAGS measurement of 45 FP nuclides had been conducted in the US in 1990's [4]. Reacent years a European group started a new series of TAGS measurement [5]. This new activity is being conducted in close contact with the International Nuclear Data Evaluation Working Party of OECD/NEA. In this paper, the US TAGS results were analyzed on the basis of the gross theory of beta decay in order to improve the prediction ability of the theory.

\section{Pandemonium and TAGS}

\subsection{Pandemounim problem}

Suppose that a highly $Q_{\beta}$-valued nucleus is decaying and the $\beta$-transitions feed the very high energy levels of the daughter nuclide. These $\beta$-feedings are tend to be overlooked in the experimental, evaluating and schematizing procedures of decay data because of the weak and complicated nature of the $\gamma$-cascades which follow the $\beta$-transitions. This situation is illustrated in figure 1 . Then the $\beta$-ray energy release per decay (or average $\beta$-ray energy, $E_{\beta}$ tends to be overestimated and the $\gamma$-ray energy release (or the average $\gamma$-ray energy, $\left.E_{\gamma}\right)$ to be underestimated. In 1977 Hardy et al. [2] argued using a computer-simulation method that this kind of missing strengths is inevitable in the complicated decay schemes

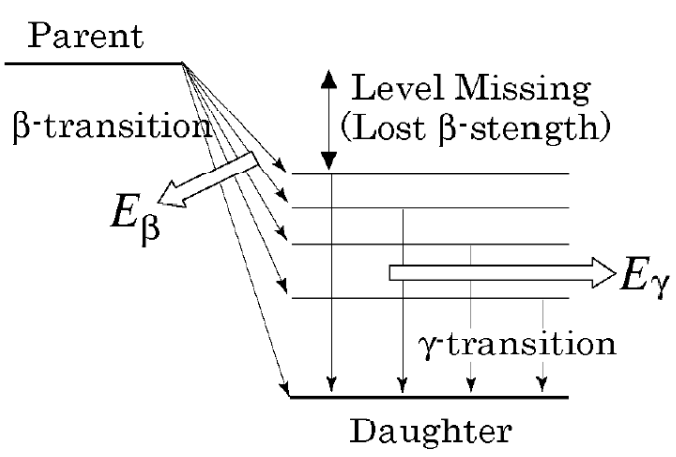

Fig. 1. Illustration of the "pandemonium" problem.

having high $Q_{\beta}$-value. Hardy et al. named the nuclide with this problem in it a pandemomium after J. Hilton's "Paradise Lost".

\subsection{Total absorption $\gamma$-ray spectroscopy}

Greenwood et al. at INEL (Idaho Nuclear Engineering Laboratory) measured the $\beta$-feeding as a function of the excitation energy in the daughter nuclide for 45 isotopes of $12 \mathrm{FP}$ elements (Rb, Sr, Y, Cs, Ba, La, Ce, Pr, Nd, Pm, Sm and $\mathrm{Eu}$ [ [4]. The $\gamma$-ray detector they used was a $25.4 \mathrm{~cm}$ diameter $\times 30.5 \mathrm{~cm}$ long $\mathrm{NaI}(\mathrm{Tl})$ scintillator installed at the INEL online mass separator. In principle all of the $\gamma$-rays emitted in a cascade accompanied by a de-excitation of a certain level deposit all of their energies into the scintillator. Thus the pulse height gives the level energy into which the preceding $\beta$-transition have taken place. These results are exactly the data required to calculate the average $\beta$ - and $\gamma$-ray energies per one $\beta$-decay of the parent FP nucleus, or $E_{\beta}$ and $E_{\gamma}$ Therefore, if this total gamma-ray absorption spectroscopy (TAGS) measurements are carried out in an ideal way, the values of $E_{\beta}$ and $E_{\gamma}$ obtained from them are free from the pandemonium problem. 


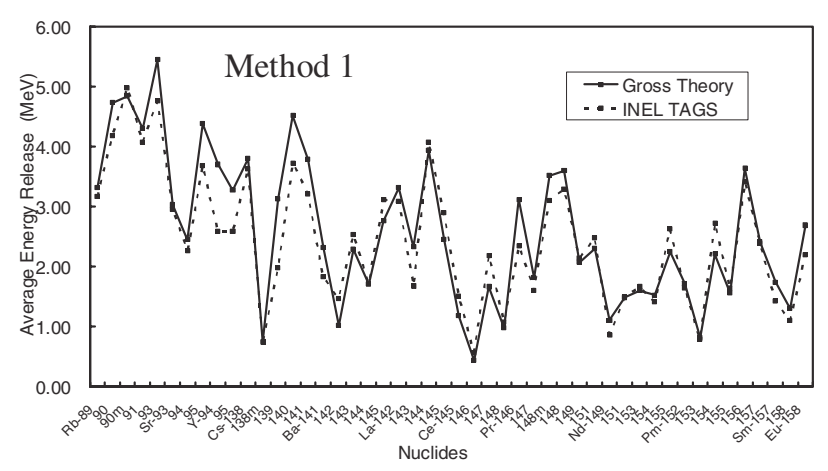

Fig. 2. INEL/TAGS and calculated total energies $E_{\beta}+E_{\gamma}$. Parameter $Q_{00}$ fixed to reproduce the measured half-life.

\subsection{Introduction of the beta-decay theory}

In order to overcome or to circumvent the pandemonium problem, Yoshida and Nakasima proposed a method to compensate these lost $\beta$-strengths on the basis of a nuclear-model calculation [6]. They applied the gross theory of $\beta$-decay developed by Takahashi and Yamada et al. [7] to correct for the pandemonium effect hiding in the preliminary version of the JNDC FP Decay Data File, which is the predecessor of the JENDL FP Decay Data File [8] (hereafter JENDL). For doing this the authors have to tune a parameter $Q_{00}$ in the theory so that the theoretical calculation reproduce the measured halflife well for the decay of each $Q_{\beta}$-value nuclide. Now we have 45 isotopes of $12 \mathrm{FP}$ elements mentioned above, of which we know the experimantal half-lives and the values of $E_{\beta}$ and $E_{\gamma}$ These values can be expected to be pandemonium-effect free as mentioned in section 2.2.

\section{Tuning the theory with TAGS data}

\subsection{Determination of model parameter}

The parameter $Q_{00}$ in the gross theory stands for the excitation energy of the lowest level accesible by the $\beta$-transition from the parent nuclide. In this sense $Q_{00}$ reflects the effect of the selection rules in the low-lying levels in the daughter nuclide. First of all we determined the $Q_{00}$ value of each of 45 nuclides introduced in section 2.2 so as to reproduce the measured halflife in the best way. In generating JENDL, this method was employed. Then, fixing the parameter as thus obtained, we calculated the released energies, $E_{\beta}$ and $E_{\gamma}$. Figure 2 compares the total energy release, $E_{\beta}+E_{\gamma}$ for each of 45 nuclides thus calculated with those reduced from the $\beta$-feeds measured by the TAGS technique. We call this method as Method 1 .

As the second method, we tried to reproduce the total energies, $E_{\beta}+E_{\gamma}$, reduced from the TAGS data by optimising the parameter $Q_{00}$. Thus we got a $Q_{00}$ value for each nuclide. Then we classified the nuclides into three groups, odd-odd, even-even, and odd $\mathrm{A}$ according to the neutron and the proton numbers, $\mathrm{N}$ and $\mathrm{Z}$, with $\mathrm{A}=\mathrm{N}+\mathrm{Z}$ and averaged the $Q_{00}$ within these three groups. The results are $0.95,0.63$, and $0.95 \mathrm{MeV}$ for the odd-odd, even-even, and odd A groups, respectively. Then using these average values, we recalculated the total

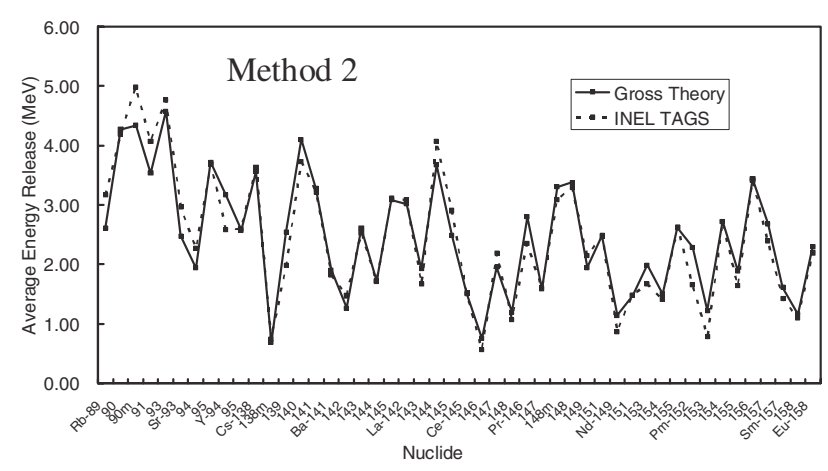

Fig. 3. INEL/TAGS and calculated total energies $E_{\beta}+E_{\gamma}$. Parameter $Q_{00}$ used is an average over o-o, e-e and odd-A groups.

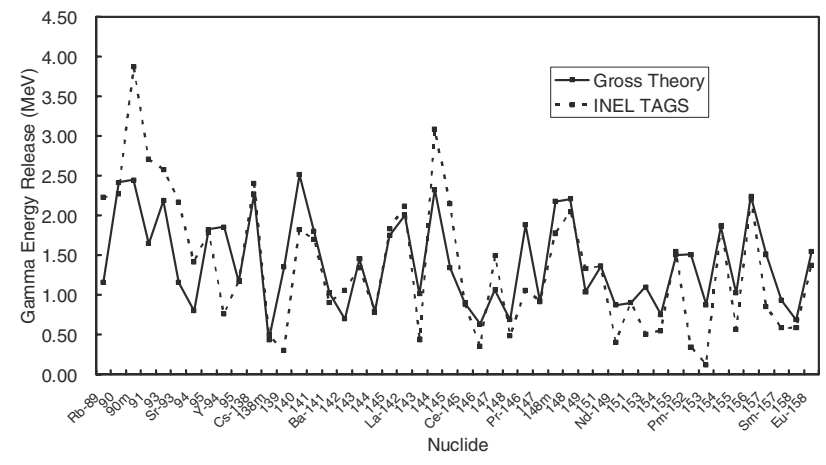

Fig. 4. Comparison between INEL/TAGS and calculated $E_{\gamma}$ with the second method, i.e., the use of the group-wise averaged $Q_{00}$.

energies, $E_{\beta}+E_{\gamma}$. We call this method as Method 2. The results are shown in figure 3.

An agreement a little better than Method 1 in terms of the $\chi$-square examination was realized by Method 2 or, in other words, by using the group-wise averages of $Q_{00}$. In generating the JNDC FP Decay Data Library [9], the ancestor of JENDL FP Decay Data File 2000 [8], Method 1 was applied for suspected pandemonium nuclides.

Practically, both in figures 2 and 3, reasonable agreement is realized for the total energy release, i.e., $E_{\beta}+E_{\gamma}$. When $E_{\beta}$ and $E_{\gamma}$ are concerned in a separate way, the situation looks fairly different. Figure 4 shows the comparison between TAGS $E_{\gamma}$ and those calculated with the second method, i.e. the use of the group-wise average of $Q_{00}$. This inconsistent result indicates that the theoretical prediction of $E_{\gamma}$ is much more difficult than that of the total energy release or $E_{\beta}+E_{\gamma}$. The situation is almost similar to this case as the average $\beta$-ray energy or $E_{\beta}$ is concerned.

\subsection{Impact on decay heat calculations}

Figures 5 and 6 show the calculated $\gamma$-ray and the $\beta$-ray components of the decay heat after a fission burst in $\mathrm{Pu}-239$, As the reference, JEFF-3.1 calculation is selected here because the $E_{\beta}$ and $E_{\gamma}$ data in JEFF-3.1 are not corrected for the pandemonium problem and, then, the effects of introducing the TAGS data or the gross theory predictions can be seen explicitly. On the other hand, JENDL is theoretically corrected for pandemonium [8] and this fact complicates the comparison. 


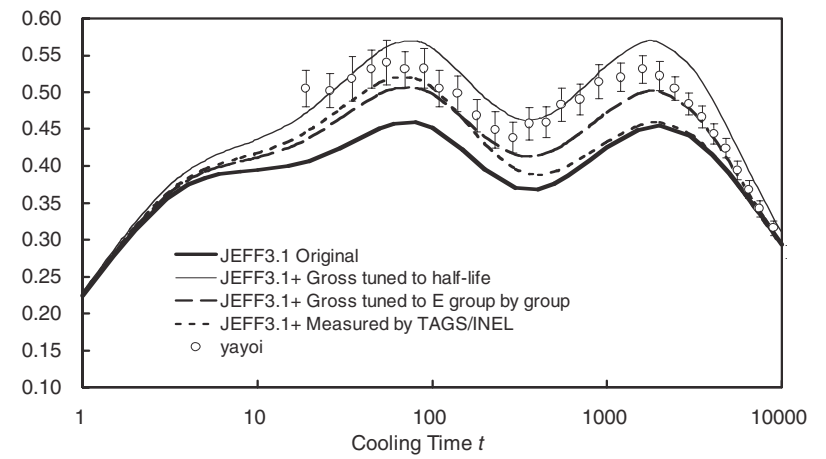

Fig. 5. Effect of introduction of INEL/TAGS and theortical prediction for FPs on the $\gamma$-ray component after a fission burst in Pu-239.

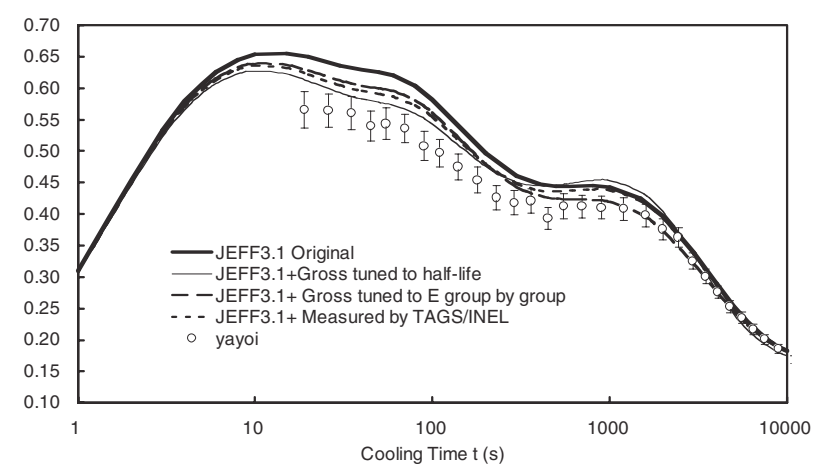

Fig. 6. Effect of introduction of INEL/TAGS and gross theory prediction for 45 FPs on the $\beta$-ray component after fission respectively.

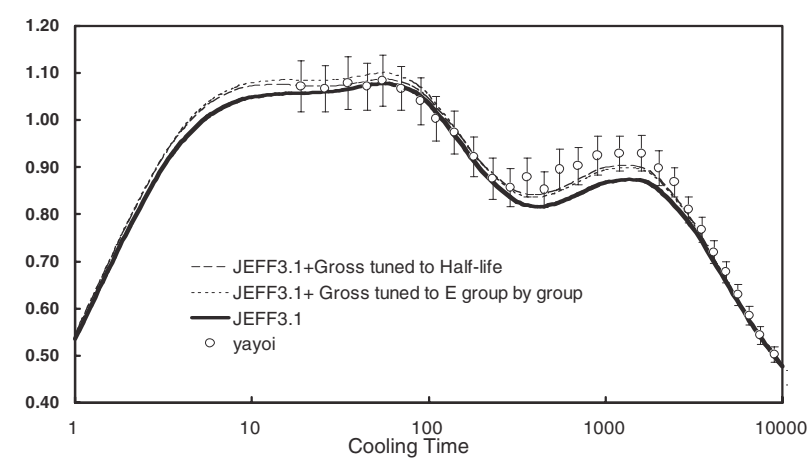

Fig. 7. Gross theory prediction of the future TAGS results for Rb-92, Y-96, Nb-98, Tc-102, Tc-104, Tc-105, Cs-142, La-145, which are among the list of the current TAGS program.

As is seen in figure 5, TAGS data improves the consistency with the Yayoi measurement [10] overall. The gross-theory prediction based on Method 2 almost follows the TAGS up to several hundred second after the fission burst.

The Method 2 prediction behaves in fairly different way. For the $\beta$-ray component shown in figure 6 , the tendency is similar to the case of the $\gamma$-ray component shown in figure 5 . But in this case, the difference is smaller than the $\gamma$-ray case.

Figure 7 is devoted to expected results from the gross theory prediction of the total energy release $\left(E_{\beta}+E_{\gamma}\right)$ for 8 nuclides, which are in the list of the candidate nuclides of the future TAGS measurement to be conducted in cooperation with the Subgroup 25 of WPEC [11]: Rb-92, Y-96, Nb-98, Tc-102, Tc-104, Tc-105, Cs-142, La-145. From this prediction, future TAGS measurements are expected to improve the reliability of the summation calculation of the FP decay heat.

\section{Conclusion}

Introduction of gross theory to summation calculation of FP decay heat succeeded in remarkably improving the agreement between summation calculations and the sample irradiation measurements for Th-232, U-233, U-235,U-238, Pu-238 and $\mathrm{Pu}-241$ without exception [8]. The theory, however, is an average theory and thus it is not suitable for predicting the decay characteristics of each individual nuclide. It is powerful when we deal with the average behavior of high Q beta-decay. On the other hand, the use of the experimental results based on the TAGS eliminates the pandemonium problem fairly well without introducing any theoretical prediction. In this paper we conclude from the comparison with the INEL/TAGS data taken in 1990's that the gross theory predicts well the total energy release, or $E_{\beta}+E_{\gamma}$ However, when $E_{\beta}$ and $E_{\gamma}$ are dealt with separately, the prediction power of the theory has a limitation. This is why we need more TAGS measurements over the fission product nuclides decaying with high-Q value. The most important implication of the gross theory is that there are sizable amount of $\beta$-strengths existing in the high excitation energy range left undetected by the conventional measurement method as far as high-Q-valued, short-lived nuclides are concerned. This is the second reason why we need more TAGS measurements.

\section{References}

1. T. Yoshida, R. Nakashima, J. Nucl. Sci. Technol. 18, 393 (1981).

2. J.C. Hardy, L.C. Carrez, B. Jonson, P.G. Hansen, Phys. Lett. 71B, 307 (1977).

3. N. Hagura, T. Yoshida, T. Tachibana, J. Nucl. Sci. Technol. 43, 497 (2006).

4. R.C. Greenwood, R.G. Helmer, M.H. Putnam, K.D. Watts, Nucl. Instrum. Meth. A 390, 95 (1997). See also R.C. Greenwood, R.G. Helmer, M.A. Lee et al., Nucl. Instrum. Meth. A 314, 514 (1992).

5. A. Algora (these proceedings).

6. T. Yoshida, R. Nakashima, J.Nucl. Sci. Technol. 18, 393 (1981).

7. K. Takahashi, M. Yamada, T. Kondoh, At. Data Nucl. Data Tables 12, 101 (1973), and references therein.

8. J. Katakura, T. Yoshida, K. Oyamatsu, T. Tachibana, JENDL FP Decay Data File 2000, JAERI 1343 Japan Atomic Energy Research Institute (2001).

9. K. Tasaka, H. Ihara, M. Akiyama et al., JNDC Nuclear Data Library of Fission Products, JAERI 1289, Japan Atomic Energy Research Institute (1983).

10. M. Akiyama, S. An, in Proc. Int. Conf. on Nuclear Data for Science and Technology, Antwerp (1982), p. 237, and references therein.

11. http://www.nea.fr/html/science/wpec/SG25/index.html. 